\title{
AADSM Accepted Late-Breaking Abstracts (2020)
}

ABSTRACT \#024

\section{OROPHARYNX 3D EVALUATION AFTER MAXILLARY EXPANSION: A COMPARISON BETWEEN TWO SOFTWARE}

Dr. Silvia Gianoni-Capenakas ${ }^{1}$, Dr. Carlos Flores-Mir², Dr. Manuel Lagravere ${ }^{3}$, Dr. Camila Pacheco-Pereira 4

${ }^{1}$ DDS, Oral Medicine Specialist, MSc, Ph.D. student in Orthodontics. School of Dentistry, Faculty of Medicine and Dentistry, University of Alberta, Canada; ${ }^{2} D D S, M S C$, Ph.D., FRCD(c), Professor \& Orthodontic Graduate, Program Director, School of Dentistry, Faculty of Medicine and Dentistry, University of Alberta, Canada. ${ }^{3} D D S, M S c$, Ph.D., FRCD(c), Associate Professor at the Orthodontic Graduate Program, School of Dentistry, Faculty of Medicine and Dentistry, University of Alberta, Canada. ${ }^{4} D D S$, MBA, MSc Dentistry, Ph.D. candidate, OMR Graduate PGY2 and Clinical Assistant Professor at the University of Texas Health Science at San Antonio \& Assistant Clinical Professor, Faculty of Medicine and Dentistry, School of Dentistry, University of Alberta, Canada.

Introduction: Maxillary expansion is used to correct maxillary deficiencies, the most common technique is the Rapid Maxillary Expansion. Several studies have reported an increase of the upper airway volume and minimal crosssectional area (MCA) after maxillary expansion, especially related to the nasal cavity and nasopharynx. However, contradictory results regarding the measured changes in the oropharyngeal portion of the upper airway have led to uncertainty about the real effect of maxillary expansion on the oropharynx dimensions.

To build the 3D model, a software is required to convert the DICOM files into 3D images. The process of converting raw CBCT data into 3D imaging its called reconstruction. The software may use different types of craniofacial segmentation using CBCTs data: manual and automatic/semi-automatic. The manual segmentation is more accurate, but also more time consuming due to the necessity to, slice by slice, delimitate the area to be included. On the other hand, in the automatic/semiautomatic method, the software automatically differentiates the air from neighboring structures according to the grey values determined as the threshold. Two greatly used software for segmentation among orthodontists are Invivo (Software A) (Anatomage, San Jose, California, US) and Dolphin (Software B) (Dolphin Imaging \& Management Solutions, Chatsworth, California US), both automatic software considered semiautomatic tools to assess upper airway segmentation. This study aimed to explore if there were differences between the measurement of two different software analyzing the oropharynx volume and MCA.

Methods: A retrospective analysis of data from a clinical trial was evaluated. Patients between 11 to 17 years old, with maxillary transverse discrepancies in need of maxillary expansion, were treated in the orthodontic clinic at the University of Alberta, Edmonton Canada. Patients underwent CBCT imaging at two time-points: T1- before treatment and after clinical evaluation, to further evaluate and assist the clinicians on the diagnosis of dental and craniofacial orthodontic discrepancies; T2- at 6 months, after maxillary expansion. The CBCT data was assessed using Software A and Software B to measure the Oropharynx volume and MCA. Statistical analysis was made at a $5 \%$ significance level $(95 \% \mathrm{CI})$ to evaluate whether or not there were changes between the two software.

Results: A pairwise comparison analysis showed a statistically significant MCA $(\mathrm{p}=0.001)$ and volume $(p<0.001)$ mean differences when comparing both software. Software B showed higher mean average results than Software A with MCA higher average $=47.533 \mathrm{~mm}^{2}$ and in Volume higher average $=1.487 \mathrm{cc}$. The large CI ranging from 0.816 to 2.158 at $95 \%$ showed low precision of the results between Software A and B.

Conclusions: Software A and B showed statistically significant different results when evaluating oropharyngeal MCA and volume; however, it was not possible to assume which one would have the most accurate measurement result when compared to the real volume and MCA in children.

Support: No external fund was used in this study

\section{ABSTRACT \#025}

INCIDENCE OF POSTERIOR OPEN BITE WITH MANDIBULAR ADVANCEMENT DEVICES IN PATIENTS WITH SLEEP RELATED BREATHING DISORDERS: A CASE CONTROL STUDY.

Redondo-deMena $\mathrm{M}^{1}$; Sánchez-Sánchez $\mathrm{T}$ 1; Moreno-Hay $1^{2}$

${ }^{1}$ Complutense University of Madrid, Spain, ${ }^{2}$ University of Kentucky, Lexington, US

Introduction: Obstructive Sleep Apnea (OSA) is caused by a complete (apnea) or partial (hypopnea) intermittently and repetitively obstruction of the Upper Airway (UA), 
leading to its collapse. The obstructive episode ends with a transient electroencephalographic micro-awakening (arousal) leading to restoration of the UA's permeability.

As a treatment option, Mandibular Advancement Devices, MAD, are used in simple snoring, mild-moderate OSA and, in recent years, severe OSA when other therapeutic alternatives have failed or the patient can not tolerate PAP therapy.

Although many articles focus on describing the effectiveness of the MAD, few evaluate the side effects. It has been estimated that the presence of posterior open bite (POB) associated with the use of MAD is around 14-18\%.

The aim of this study was to evaluate the incidence of $\mathrm{POB}$ in patients with OSA managed with MAD in a one year follow up.

Methods: Patients with OSA that were referred for therapy with MAD were recruited. A Herbst appliance was delivered and titrated until resolution of symptoms or maximal anatomical protrusion was achieved. Follow up visits were established at $4,15,25$ and 54 weeks. The control group received PAP therapy with a nasal mask and were followed up at one year.

The occlusal examination was performed using Arti-fol paper; a sheet of metallic polyester (Shimstock-Foil) 12 microns thick. POB was defined as absence of contacts in premolar and molar region, with the possibility of taking off the paper when patient closed in maximum intercuspation. Two factor ANOVAs, Pearson's correlations and Pearson's $\chi 2$ test were performed.

Results: 80 patients were recruited: 42 received a MAD and 38 PAP therapy. Six patients abandoned the study in the MAD group $(n=36)$ and 13 in the control group $(n=25)$. At subjective level $16.7 \%$ of patients in MAD group noted, bite changes at 54 weeks, of which only $2.8 \%$ noted it all the time.

On examination, the average of posterior teeth without contact increased in MAD group, from 0.71 to 2.08, and slightly decreased in PAP group from 0.32 to 0.08 , however, these differences were not significant between groups. In both groups the main effect of time was marginally significant $(\mathrm{p}<0.10)$ and in MAD group appearance of posterior open bite, POB, was observed, $(p<0.05)$ throughout the visits.

To determine the number of patients who developed POB, the variable was dichotomized (those patients with no $(0)$ pieces marked as absence of dental contact did not develop POB and those with 1 or more marked pieces developed it.) In MAD group it increased by $14 \%$ while in the PAP group the increase was smaller, $1.3 \%$.
Conclusions: The use of MAD can cause POB after one year of use, compared to PAP, and that it increases significantly over time.

Support: None

\section{ABSTRACT \#026}

\section{CORRELATION BETWEEN EXPERIMENTAL DIAGNOSIS OF SLEEP BRUXISM AND OBSTRUCTIVE SLEEP APNEA: A PILOT STUDY}

Yanez-Regonesi, F, Moreno-Hay, I.

Orofacial Pain Center, University of Kentucky, Lexington, USA.

Introduction: As suggested by a group of experts, bruxism represents a repetitive masticatory muscle activity characterized by clenching or grinding of the teeth and/or bracing or trusting of the mandible; specifying that we can have awake and sleep bruxism. The latter, has presented more controversy and we have less knowledge about it. Most recently, the same group provided an update clarifying sleep bruxism (SB) as sleep related masticatory muscle activity characterized by rhythmic (phasic) and non-rhythmic (tonic) bursts. Recently, interest has grown relating SB with other sleep disorders such as insomnia, sleep epilepsy, REM behavior disorder and sleep related breathing disorder (SRBD). SRBD represents a group of disorders including snoring, obstructive sleep apnea and upper airway resistance syndrome. Currently there is no evidence to support the association or causality between SB and SRBD. However, several studies have reported higher risk of SB in patients with obstructive sleep apnea or with snoring symptoms. It has even been suggested a protective role of $\mathrm{SB}$, with the masseter contraction occurring at the end of the apneic event. The aim of this study was to evaluate is there is co-existence of sleep bruxism as diagnosed with portable device in patient diagnosed with obstructive sleep apnea.

Methods: Patients underwent a full laboratory polysomnographic study to evaluate for obstructive sleep apnea. During that same night we evaluated for selfreported sleep bruxism with the questionnaire validated by the AADSM. Additionally, the patients were evaluated for experimental diagnosis of sleep bruxism with a validated portable device unit. Pearson correlation analysis was performed to evaluate the relationship between selfreported and experimental sleep bruxism with positive diagnoses of obstructive sleep apnea.

Results: From the 20 patients recruited, 7 were diagnosed with sleep bruxism based on the results of the portable device and 10 were diagnosed with obstructive sleep apnea based on the laboratory polysomnography. No correlation was found between the experimental diagnosis of sleep 
bruxism and self-reported bruxism, and diagnosis of obstructive sleep apnea (P: 0.11, -0.105 respectively).

Conclusion: Based on the results of this pilot study, no relationship was found between the experimental diagnosis obtained with the portable device and positive diagnosis of obstructive sleep apnea. Further research is needed to contrast the results of the portable device with those of the laboratory polysomnography.

Support: None.

\section{ABSTRACT \#027}

\section{CORRELATION BETWEEN THE SUBJECTIVE SYMPTOMS AND OBJECTIVE MEASUREMENTS IN THE MANAGEMENT OF OBSTRUCTIVE SLEEP APNEA WITH MANDIBULAR ADVANCEMENT DEVICE}

Yanez-Regonesi, F., Eisa, E., Jayaraman, S., MorenoHay, I.

Orofacial Pain Clinic, University of Kentucky, Lexington, USA.

Introduction: Obstructive sleep apnea (OSA) is a serious health condition that affects $9 \%-28 \%$ of women and $24 \%$ $26 \%$ of the US population, making it the second most diagnosed respiratory condition after asthma. Mandibular advancement devices (MAD) is an effective treatment option for the management of mild to severe obstructive sleep apnea. A third of OSA patients treated with MAD therapy show a reduction in the apnea-hypopneas index (AHI) to $<5 / \mathrm{h}$, and another third will have a $>50 \%$ reduction in AHI, while a third will not achieve the $50 \%$ mark. Titration protocol is generally based on the patient's subjective improvement in daytime sleepiness, quality of sleep, and report of reduction/elimination of snoring and witnessed apneas. In fact, several clinical trials have reported the efficacy of the appliance in improving the patient's daytime sleepiness and quality of life. However, due to time constraints, limited resources, or a physician's decision, sometimes a second sleep study to assess the efficacy of the oral appliance is not performed. The aim of this study was to establish if there is any correlation between subjective symptoms and objective results obtained from the follow-up sleep study.

Methods: Participants referred to the Orofacial Pain Clinic for the treatment of obstructive sleep apnea with mandibular advancement devices were evaluated for subjective symptoms of improvement. Once the patient reported a significant improvement in sleep quality or maximum anatomical capacity was reached, they were referred to their sleep physician for a follow-up sleep study. Correlation analysis with Pearson correlation test was performed to evaluate if there is any relationship between the subjective symptoms reported by the patients upon referral to a second sleep study and the baseline and follow up AHI. For analysis, two different criteria's for improvement in obstructive sleep apnea were established. The first criteria was set at $50 \%$ improvement in the AHI. The second criteria was set at $50 \%$ improvement in the AHI, with AHI below 10 .

Results: Fifty-seven patients completed a follow-up sleep study. $84.2 \%$ of the patients reported $70 \%$ or more improvement in their symptoms upon referral for a followup sleep study. Follow up studies were performed by means of polysomnography, home sleep study, or pulseoximetry in $19.3 \%, 50.88 \%$, and $29.82 \%$, respectively. No significant changes were seen in the weight (pounds) at baseline and follow up. AHI was reduced by $50 \%$ in $50.8 \%$ of the patients, and in $40.35 \%$ of patients the AHI was below 10.Hence, no correlation was seen between subjective symptoms and the results from the follow-up sleep study with criteria 1 or 2 (P: $0.172,0.160$ respectively)

Conclusions: Based on the results of this study, subjective symptoms of improvement in sleep quality does not predict an improvement in AHI with the use of MAD. Therefore, the trained dentist should recommend their patient to perform a follow-up sleep study to assess the effectiveness of their oral appliance despite self-reported improvement of patient's symptoms.

\section{Support: None}

\section{ABSTRACT \#028}

\section{USING APNEA BURDEN AS AN INDICATOR OF DISEASE RELIEF IN OSA PATIENTS AND AS A TOOL FOR EVALUATING THE SUCCESS RATE OF TWO APPLIANCES.}

\section{${ }^{1}$ Erika Mason DDS, D-ABDSM, D-ACSDD, ${ }^{2}$ David Kuhns PhD}

\section{${ }^{1}$ Sleep Better Virginia, ${ }^{2}$ ProSomnus Sleep Technologies}

Introduction: The Apnea/Hypopnea Index (AHI) is the primary metric for establishing a successful treatment, but too often the changes or lack of change in the AHI do not correspond to the reduction in symptoms or improvement in health of the patient. This study digs deeper into AHI, the shift of apneas to hypopneas and the overall reduction in apnea burden on the patient. This evaluation also provides insight for the clinician as to why similar AHI levels may also have variance in the Oxygen desaturation metrics. Additionally, will the design of an appliance impact the apnea burden differently? Two appliances were tested, a precision CAD/CAM device and a traditionally hand-made device.

Methods: This single center study utilized the existing patient pool of the dental practice to be treated with the ProSomnus $[\mathrm{PH}]$ Sleep appliance (ProSomnus Sleep Technologies, Pleasanton CA) and retroactively looked at 
cases previously treated with the Somnodent Herbst Advanced, "Herbst", (Somnomed, Plano, TX). Patients were evaluated consecutively and had PSG and/or a one night HST data in order to access apnea and hypopnea durations. 32 patients were evaluated, 4 patients were lost to follow up. Patients had an average AHI of $14.9+/-6.5$, (9 males, 6 females), ages of $66.7+/-9.0$ for the $[\mathrm{PH}]$ appliance and AHI of 14.2 +/- 9.0, (7 males, 6 females), ages of $63.7+/-10.7$ for the Herbst appliance. All patients had refused or discontinued CPAP and were instructed to use elastics with their appliance. Apnea-hypopnea burden was calculated as the \% time of sleep for an apneic/hypopneic event greater than $10 \mathrm{~s}$ using the $4 \%$ desaturation criteria.

Results: Both devices demonstrated a significant change in AHI from pre to post treatment, the $[\mathrm{PH}]$ had a reduction of AHI of 6.9 and the Herbst 7.3, the difference between the two appliances was not significant in terms of AHI, however, the $[\mathrm{PH}]$ appliance did show a significant reduction in apnea burden with a mean change of $5.9 \%$ compared to the Herbst of only $2.3 \%$ with a p-value of 0.049 , indicating a larger impact on disease reduction for the $[\mathrm{PH}]$ appliance. The $[\mathrm{PH}]$ also showed a larger improvement in O2 DSAT of $3.5 \%$ compared to $2.0 \%$ for the Herbst. Subjects using $[\mathrm{PH}]$ devices went from a mean apea/hypopnea ratio of 1.8 (pre-score) to a post mean ratio of 0.45 , a decrease of $75 \%$ (p-value 0.04). Subjects using Herbst devices did not significantly decrease the apea/hypnea ratio ( $\mathrm{p}$-value 0.42 ).

Conclusions: Even though the reduction in AHI was similar for both devices, the $[\mathrm{PH}]$ device showed a significantly better reduction in apnea burden and oxygen desaturation than the Herbst. One possible reason for this difference could be attributed to the significant difference in bulk of the two devices, especially the minimal lingual bulk of the $[\mathrm{PH}]$ device.

Support: This study was supported by ProSomnus Sleep Technologies and the staff at Sleep Better Virginia

\section{ABSTRACT \#029}

\section{AN EVALUATION OF THE STARTING BITE POSITION IN CUSTOM ORAL APPLIANCE THERAPY (OAT) DEVICES: ARE OAT DEVICE ACCURACY AND PRECISION AS EXPECTED?}

Jerry $\mathrm{Hu}^{1}$ DDS, D-ABDSM, D-ASBA, D-ACSDD, Len Liptak $^{2}$ MBA, Sung Kim²

\section{${ }^{1}$ Smiles of Alaska; ${ }^{2}$ ProSomnus Sleep Technologies}

Introduction: Variability exists in manufacturing, whether fabricating neurovascular stents or OAT devices. "Accuracy" describes the extent that a manufactured item conforms to a target. For OAT, clinically significant starting bite accuracy errors may impact treatment effectiveness, side effects, and clinical efficiency. Further, device titration may amplify starting bite accuracy errors. "Precision" measures the errors introduced when repeating a process. Precision is associated with predictable starting bite performance.

To the best of the authors' knowledge, this is the first study to evaluate the accuracy and precision of the starting bite (SB) positions of custom OAT devices relative to the target bite (TB) provided by the dental sleep medicine provider.

Methods: Multiple sets of four different types of OAT devices $(n=11)$ were fabricated from the same set of digital records to control variability. The custom OAT device types were: Nylon Strap (NS) n=3, Precision Manufactured (PM) $n=3$, Reverse Dorsal (RD) $n=3$, and Traditional Dorsal (TD) $n=2$. (Note, Fulcrum Strap type OAT devices were ordered but were not delivered in time.)

Accuracy and precision (Anterior/Posterior, Vertical and Lateral) were determined by overlaying the digitized dental cast positions at the SB locations for each OAT device against the baseline digitized dental cast position at the TB. Measurements were made in CAD software to mitigate the risk of experiment error.

Based on e 0486 coding, AADSM practice guidelines and published papers, $1 \mathrm{~mm}$ was adopted as the threshold for clinical significance.

Results: The average total accuracy error between the TB and the SB was $1.63 \mathrm{~mm}$ across all eleven devices and exceeded the threshold for clinical significance. The average $\mathrm{A} / \mathrm{P}$ accuracy error was clinically significant at $1.07 \mathrm{~mm}$. The average vertical and lateral accuracy errors were $0.65 \mathrm{~mm}$ and $0.42 \mathrm{~mm}$ respectively.

Eight of eleven devices tested had total starting bite accuracy errors that exceeded the $1 \mathrm{~mm}$ threshold for clinical significance. PM type OAT devices exhibited the lowest average accuracy error at $0.32 \mathrm{~mm}$ and were the only device type that did not have a clinically significant accuracy error. The TD OAT device type exhibited the largest average accuracy error at $3.74 \mathrm{~mm}$.

The average precision error for all devices tested was $0.70 \mathrm{~mm}$. PM type OAT devices demonstrated the best precision at $0.29 \mathrm{~mm}$. The RD type OAT devices demonstrated the largest total precision error at $1.26 \mathrm{~mm}$.

Conclusions: Clinically significant starting bite accuracy errors were observed in all devices except the PM type. Clinically significant errors may impact treatment effectiveness, side effects and clinical efficiency.

A wide range of precision errors were quantified across the four different OAT device types. Certain OAT device types will deliver more predictable starting bites than others.

Support: Support provided by ProSomnus Sleep Technologies. The authors would like to thank Jerry Simon 
and Majid Ghaderiardakani of ProSomnus Sleep Technologies and Rebecca Kompkoff of Smiles of Alaska for supporting this project.

\section{ABSTRACT \#030}

\section{AN EVALUATION OF CUSTOM ORAL APPLIANCE (OAT) DEVICE VOLUMES}

Jerry $\mathrm{Hu}^{1}$ DDS, D-ABDSM, D-ASBA, D-ACSDD, Len Liptak ${ }^{2}$ MBA

\section{${ }^{1}$ Smiles of Alaska, ${ }^{2}$ ProSomnus Sleep Technologies}

Introduction: Does OAT device volume matter? Are there meaningful differences in OAT device volumes based on device type? It is thought that OAT device volume may correlate with patient comfort and treatment adherence. Adherence is a limitation of PAP therapy. Device (dis)comfort is cited by physicians as a reservation about prescribing OAT. OAT device volume may also impact tongue space, tongue posture, the degree of mandibular protrusion and the expression of intraoral exostoses, which can impact treatment efficacy and side effects.

Additionally, patients and dental sleep providers often use volume and available tongue space as criterion for device selection. This can be challenging, however. The device models from various manufacturers are made on different size dental casts.

To the best of the authors' knowledge, this is the first study to objectively quantify the overall volume (in $\mathrm{mm}^{\wedge} 3$ ) of different types of custom oral appliance therapy devices.

Methods: Multiple sets of five different types of custom OAT devices $(n=11)$ were ordered from the same set of digital records. The custom OAT device types were: Nylon Strap (NS) $n=3$, Precision Manufactured (PM) $n=3$, Reverse Dorsal (RD) $\mathrm{n}=3$, and Traditional Dorsal (TD) $\mathrm{n}=2$.

The displacement method was utilized to calculate device volume. Each OAT device was submerged into a graduated cylinder with a known volume of water. The amount of water displaced by each OAT device was measured to calculate volume. Three cycles of displacement measurements were made for each device to control for measurement error.

Results: The average volume for all OAT devices tested in this evaluation was $2.50 \mathrm{~mm}^{\wedge} 3+/-0.88$. The single lowest volume device was $1.26 \mathrm{~mm}^{\wedge} 3$. The largest volume device was $3.82 \mathrm{~mm}^{\wedge} 3$. The largest device was 3.04 times more voluminous than the smallest device.

Amongst the five device types tested, the Precision Manufactured type devices had the smallest average volume of $1.41 \mathrm{~mm}^{\wedge} 3+/-0.14$. The Traditional Dorsal type devices had the largest average volume of $3.81 \mathrm{~mm}^{\wedge} 3+/-$ 0.03 . The Nylon Strap type device had an average volume of $2.23 \mathrm{~mm}^{\wedge} 3,+/-0.21$. The Reverse Dorsal type devices had an average volume of $2.99 \mathrm{~mm}^{\wedge} 3+/-0.11$.

Based on a visual inspection of the device types, the differences in volume appear in two design dimensions: the lingual aspect of the splint and the titration mechanism.

Conclusions: The volumes of custom OAT devices vary, significantly, by device type. Clinicians may wish to consider the actual device volume, and the related clinical implications, when selecting and prescribing a custom OAT device.

Support: Funding for sample devices provided by ProSomnus Sleep Technologies. The authors would like to thank Michelle Bryant of ProSomnus Sleep Technologies and Rebecca Kompkoff of Smiles of Alaska for supporting this project. 